\title{
Synthesis and X-ray structure of the
}

\author{
$\left(\mathrm{py}_{2}(\mathrm{NMe})_{2}\right) \mathrm{Mn}^{\mathrm{II}} \mathrm{Cl}_{2}$ and $\left[\left(\mathrm{py}_{2}(\mathrm{NMe})_{2}\right) \mathrm{Mn}^{\mathrm{III}} \mathrm{F}_{2}\right]^{+}$ \\ complexes $\left(\mathrm{py}_{2}(\mathrm{NMe})_{2}=N, N^{\prime}\right.$-dimethyl-2,11-
}

diaza[3,3](2,6)pyridinophane). High-field EPR and

DFT study of the Mn(III) complex. Evidence for a

low lying spin triplet state.

Belén Albela ${ }^{a}$, Riccardo Carina ${ }^{a}$, Clotilde Policar $^{b}$, Sandrine Poussereau ${ }^{a}$, Joan CanoBoquera $^{a, e}$, Jean Guilhem ${ }^{c}$, Luba Tchertanov ${ }^{c}$, Geneviève Blondin $^{a}$, Michel Delroisse $^{d}$, JeanJacques Girerd*a

a) Laboratoire de Chimie Inorganique, UMR 8613, Institut de Chimie Moléculaire et des Matériaux d'Orsay, Université Paris-Sud, F-91405 Orsay, France; b) Laboratoire de Chimie Bioorganique et Bioinorganique, UMR 8124, Institut de Chimie Moléculaire et des Matériaux d'Orsay, Université Paris-Sud, F-91405 Orsay, France; c) Laboratoire de Cristallochimie, Institut de Chimie des Substances Naturelles, UPR 2301, F-91198 Gifsur-Yvette, France; d) Unilever Research Port Sunlight Laboratory, Wirral, L63 3JW, UK;

e) Departament de Química Inorgànica and Centre de Recerca en Química Teórica, Universitat de Barcelona, Av. Diagonal 647, 08027 Spain. Institució Catalana de Recerca i Estudis Avançats (ICREA). 
E-mail: jjgirerd@icmo.u-psud.fr

Supporting Information



Figure S1. Chain structure of $\mathbf{2}\left(\mathrm{PF}_{6}\right)$ running along the $\mathrm{b}$ axis with the separations between the pyridine centroids as well as the $\mathrm{C} 4 \ldots \mathrm{F}$ and $\mathrm{C}^{\prime}$ '...F' distances in $\AA$.

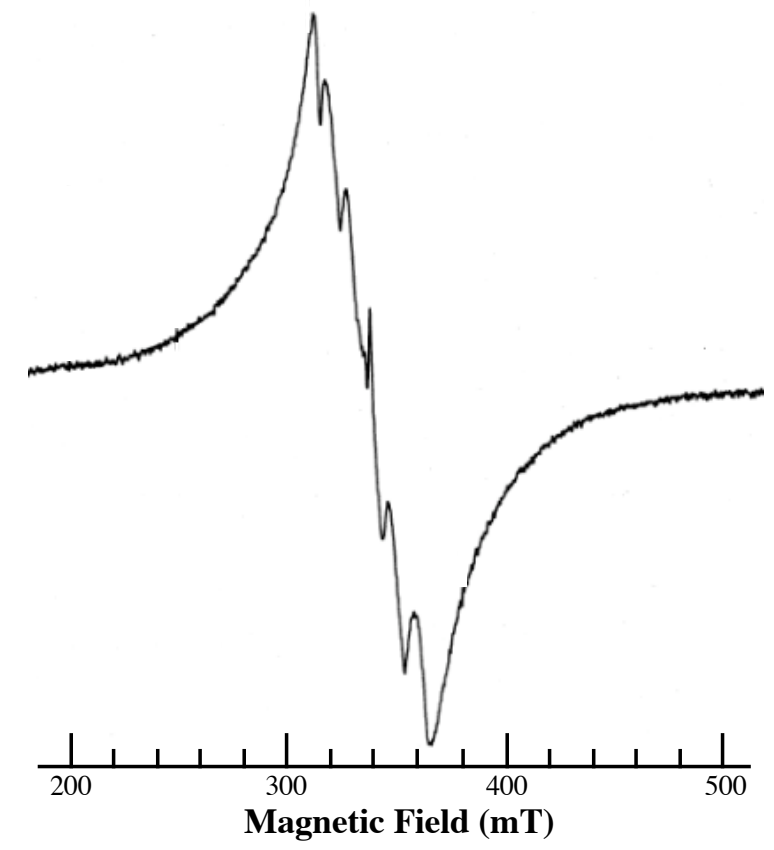

Figure S2. X-band EPR spectrum recorded on a frozen acetonitrile solution of 1. Recording conditions: $v=9.5076 \mathrm{GHz}$, modulation frequency $=100 \mathrm{kHz}$, modulation amplitude $=2 \mathrm{G}$, microwave power $=12.5 \mathrm{~mW}, T=110 \mathrm{~K}$. 
A first evaluation of the zero-field splitting tensor and of the $g$-matrix can be performed by considering the influence of the excited spin states issued from the same free ion spectroscopic term as the ground state. For an orbitally non-degenerate ground state and using the Russell-Saunders Hamiltonian for the spin-orbit coupling, one has:

$$
\begin{aligned}
& {[g]=g_{e}\left[I d_{3}\right]-2 \lambda[\Lambda]} \\
& {[D]=-\lambda^{2}[\Lambda]}
\end{aligned}
$$

where $g_{e},\left[I d_{3}\right]$, and $\lambda$ are the free electron Landé factor, the $3 \times 3$ identity matrix, and the spin-orbit coupling constant associated with the free ion spectroscopic term, respectively. The elements of the matrix $[\Lambda]$ can be calculated according to $(\mathrm{i}, \mathrm{j}=x, y, z)$ :

$$
\Lambda_{i j}=\sum_{n} \frac{\left\langle\psi_{g}\left|\hat{L}_{i}\right| \psi_{n}\right\rangle\left\langle\psi_{n}\left|\hat{L}_{j}\right| \psi_{g}\right\rangle}{E_{0}\left(\psi_{n}\right)-E_{0}\left(\psi_{g}\right)}
$$

where $\psi_{g}$ and $\psi_{n}$ are the ground and excited spin states, respectively.

In the present case, the free ion spectroscopic term is ${ }^{5} \mathrm{D}$ and the ${ }^{5} \mathrm{~B}_{1}$ ground state in $C_{2 v}$ symmetry is associated with a vacant $d_{x y}$ orbital. The two ${ }^{5} \mathrm{~A}_{1}$ states can admix and the mixing parameter $\theta$ is defined as:

$$
\begin{aligned}
& \left|1^{5} A_{1}\right\rangle=\cos \theta\left|d_{z^{2}}\right\rangle+\sin \theta\left|d_{x^{2}-y^{2}}\right\rangle \\
& \left|2^{5} A_{1}\right\rangle=-\sin \theta\left|d_{x^{2}-y^{2}}\right\rangle+\cos \theta\left|d_{z^{2}}\right\rangle
\end{aligned}
$$

where only the vacant $\mathrm{d}$ orbital is indicated. With these definitions, one can establish that the $\Lambda$-matrix is diagonal in the $(x, y, z)$ axis system drawn on Scheme 2 with the following principal components:

$$
\begin{aligned}
& \Lambda_{x x}=\frac{1}{E_{0}\left({ }^{5} B_{2}\right)-E_{0}\left({ }^{5} B_{1}\right)}=\frac{1}{\Delta E_{x y \rightarrow x z}} \\
& \Lambda_{y y}=\frac{1}{E_{0}\left({ }^{5} A_{2}\right)-E_{0}\left({ }^{5} B_{1}\right)}=\frac{1}{\Delta E_{x y \rightarrow y z}}
\end{aligned}
$$




$$
\Lambda_{z z}=\frac{4 \sin ^{2} \theta}{E_{0}\left(1^{5} A_{2}\right)-E_{0}\left({ }^{5} B_{1}\right)}+\frac{4 \cos ^{2} \theta}{E_{0}\left(2^{5} A_{2}\right)-E_{0}\left({ }^{5} B_{1}\right)}
$$

The associated d-d hole transition has been indicated except for $\Lambda_{z z}$. For $\theta=0$, one has $\Lambda_{z z}=\frac{4}{E_{0}\left(2^{5} A_{2}\right)-E_{0}\left({ }^{5} B_{1}\right)}=\frac{4}{\Delta E_{x y \rightarrow x^{2}-y^{2}}}$ while for $\theta=90^{\circ}, \Lambda_{z z}=\frac{4}{E_{0}\left(1^{5} A_{2}\right)-E_{0}\left({ }^{5} B_{1}\right)}=\frac{4}{\Delta E_{x y \rightarrow z^{2}}}$

Consequently, the $g$-matrix is:

$$
[g]=\left(\begin{array}{ccc}
g_{x} & 0 & 0 \\
0 & g_{y} & 0 \\
0 & 0 & g_{z}
\end{array}\right)=\left(\begin{array}{ccc}
g_{e}-2 \lambda \Lambda_{x x} & 0 & 0 \\
0 & g_{e}-2 \lambda \Lambda_{y y} & 0 \\
0 & 0 & g_{e}-2 \lambda \Lambda_{z z}
\end{array}\right)
$$

This allows to establish Eq. 1a-c given in the main text.

$$
\begin{aligned}
& g_{x}=g_{e}-\frac{2 \lambda}{E_{0}\left({ }^{5} B_{2}\right)-E_{0}\left({ }^{5} B_{1}\right)} \\
& g_{y}=g_{e}-\frac{2 \lambda}{E_{0}\left({ }^{5} A_{2}\right)-E_{0}\left({ }^{5} B_{1}\right)} \\
& g_{z}=g_{e}-8 \lambda\left\{\frac{\sin ^{2} \theta}{E_{0}\left(1^{5} A_{1}\right)-E_{0}\left({ }^{5} B_{1}\right)}+\frac{\cos ^{2} \theta}{E_{0}\left(2^{5} A_{1}\right)-E_{0}\left({ }^{5} B_{1}\right)}\right\}
\end{aligned}
$$

Similarly, the zero-field splitting tensor expresses as:

$$
[D]=\left(\begin{array}{ccc}
-\lambda^{2} \Lambda_{x x} & 0 & 0 \\
0 & -\lambda^{2} \Lambda_{y y} & 0 \\
0 & 0 & -\lambda^{2} \Lambda_{z z}
\end{array}\right)
$$

From the expressions of the $\Lambda$-matrix elements, it can be seen that $\Lambda_{z z}>\Lambda_{x x}, \Lambda_{y y}$, whatever the value of $\theta$. Consequently, the principal distortion axis of the ZFS tensor is aligned along the $z-$ axis and one gets:

$$
[D]=\left(\begin{array}{ccc}
-\frac{1}{3} D+E & 0 & 0 \\
0 & -\frac{1}{3} D-E & 0 \\
0 & 0 & \frac{2}{3} D
\end{array}\right)
$$


This leads to the following expressions of the $D$ and $E$ parameters, given in Eq. 1d-e in the main text:

$$
\begin{aligned}
& D=-\lambda^{2}\left\{\Lambda_{z z}-\frac{1}{2}\left(\Lambda_{x x}+\Lambda_{y y}\right)\right\}=\frac{\lambda}{2}\left\{g_{z}-\frac{g_{x}+g_{y}}{2}\right\} \\
& E=-\frac{\lambda^{2}}{2}\left(\Lambda_{x x}-\Lambda_{y y}\right)=\frac{\lambda}{4}\left\{g_{x}-g_{y}\right\}
\end{aligned}
$$

Because of the $\mathrm{d}^{4}$ configuration, the spin-orbit constant $\lambda$ is positive. The $D$-parameter is thus negative. 